\title{
Face Recognition Using Some Aspects of the Infant Vision System and Associative Memories
}

\author{
Roberto A. Vazquez, Humberto Sossa, and Beatriz A. Garro \\ Centro de Investigación en Computación - IPN \\ Av. Juan de Dios Batiz, esquina con Miguel de Othon de Mendizábal \\ Ciudad de México, 07738, México \\ ravem@ipn.mx, hsossa@cic.ipn.mx, bgarrol@ipn.mx
}

\begin{abstract}
Several associative memories (AM) have been proposed in the last years. These AMs have several constraints that limit their applicability in complex problems such as face recognition. Despite of the power of these models, they cannot reach its full power without applying new mechanisms based on current and future studies on biological neural networks. In this research we show how a network of dynamic associative memories (DAM) combined with some aspects of the infant vision system could be efficiently applied to the face recognition problem. Through several experiments by using a benchmark of faces the accuracy of the proposal is tested.
\end{abstract}

Keywords: Associative memories, face recognition, infant vision system.

\section{Introduction}

Several statistical computationally expensive techniques such as principal component analysis [2] and factor analysis have been proposed for solving the face recognition problem. These techniques have been combined with neural networks and other classifiers. Although the accuracy of these methods is acceptable, they are computationally expensive techniques. On the other hand, several associative memories (AM) have been proposed in the last years [1], [7], [16] and [17]. These AMs have several constraints that limit their applicability in complex problems such as face recognition. Despite of the power of these AMs, they cannot reach their full power without applying new mechanisms based on current and future studies on biological neural networks. In this research we described how a network of dynamic associative memories (DAM) combined with some aspects of the infant vision system could be applied to the face recognition problem. Through several experiments with a benchmark of faces the accuracy of the proposal is tested.

\section{Some Aspects of the Infant Vision System}

Vision has been a popular subject of study, but most of the research has focused on the fully developed visual systems of adult humans. To understand the role of the brain in perceptual processes such face recognition, we propose to focus on the visual 
system's development from infancy to adulthood. Within a few months of birth, the brain can differentiate faces from other faces or objects. Indeed, an infant can recognize a known face from a stranger's.

Some current researches see for example [11] and [13], have demonstrated that young babies are not capable of detecting subtle features in a face. This fact could suggest that only few information of the face is used by the baby for learning and recognition. However, the question remains: which information the baby uses to recognize a face?

In this research, we conjecture that babies extract the information of a face at random and then use this information for further recognition. We call this information stimulating points SPs.

\section{Dynamic Associative Model}

This model is not an iterative model as Hopfield's model [1]. The model emerges as an improvement of the model proposed in [9] which is not an iterative model and the results are presented in [18]. Let $\mathbf{x} \in \mathbf{R}^{n}$ and $\mathbf{y} \in \mathbf{R}^{m}$ an input and output pattern, respectively. An association between input pattern $\mathbf{x}$ and output pattern $\mathbf{y}$ is denoted as $\left(\mathbf{x}^{k}, \mathbf{y}^{k}\right)$, where $k$ is the corresponding association. Associative memory $\mathbf{W}$ is represented by a matrix whose components $w_{i j}$ can be seen as the synapses of the neural network. If $\mathbf{x}^{k}=\mathbf{y}^{k} \forall k=1, \ldots, p$ then $\mathbf{W}$ is auto-associative, otherwise it is hetero-associative. A distorted version of a pattern $\mathbf{x}$ to be recalled will be denoted as $\tilde{\mathbf{x}}$. If an associative memory $\mathbf{W}$ is fed with a distorted version of $\mathbf{x}^{k}$ and the output obtained is exactly $\mathbf{y}^{k}$, we say that recalling is robust.

\subsection{Building the Associative Memory}

The brain is a dynamic, changing neural network that adapts continuously to meet the demands of communication and computational needs [8]. This fact suggests that some connections of the brain could change in response to some input stimuli. We humans, in general, do not have problems to recognize patterns even if these appear altered by noise. Several parts of the brain interact together in the process of learning and recalling a pattern, for details refer to [3] and [6]. Based on the above example we have defined in our model several interacting areas, one per association we would like the memory to learn. Also we have integrated the capability to adjust synapses in response to an input stimulus.

Before an input pattern is learned or processed by the brain, it is hypothesized that it is transformed and codified by the brain. In our model, this process is simulated using the following procedure recently introduced in [10]:

Procedure 1. Transform the fundamental set of associations into codified patterns and de-codifier patterns:

Input: FS Fundamental set of associations:

\{1. Make $d=$ const and make $\left(\overline{\mathbf{x}}^{1}, \overline{\mathbf{y}}^{1}\right)=\left(\mathbf{x}^{1}, \mathbf{y}^{1}\right)$ 
2. For the remaining couples do \{

For $k=2$ to $p$ \{

$$
\begin{aligned}
\text { For } i & =1 \text { to } n\{ \\
\bar{x}_{i}^{k} & =\bar{x}_{i}^{k-1}+d ; \quad \hat{x}_{i}^{k}=\bar{x}_{i}^{k}-x_{i}^{k} ; \bar{y}_{i}^{k}=\bar{y}_{i}^{k-1}+d ; \quad \hat{y}_{i}^{k}=\bar{y}_{i}^{k}-y_{i}^{k}
\end{aligned}
$$

3)\} Output: Set of codified and de-codifying patterns.

This procedure allows computing codified patterns from input and output patterns denoted by $\overline{\mathbf{x}}$ and $\overline{\mathbf{y}}$ respectively; $\hat{\mathbf{x}}$ and $\hat{\mathbf{y}}$ are de-codifying patterns. Codified and decodifying patterns are allocated in different interacting areas and $d$ defines of much these areas are separated. On the other hand, $d$ determines the noise supported by our model. In addition a simplified version of $\mathbf{x}^{k}$ denoted by $s_{k}$ is obtained as:

$$
s_{k}=s\left(\mathbf{x}^{k}\right)=\operatorname{mid} \mathbf{x}^{k}
$$

where mid operator is defined as $\mathbf{m i d} \mathbf{x}=x_{(n+1) / 2}$.

When the brain is stimulated by an input pattern, some regions of the brain (interacting areas) are stimulated and synapses belonging to those regions are modified.

In our model, the most excited interacting area is call active region (AR) and could be estimated as follows:

$$
\operatorname{ar}=r(\mathbf{x})=\arg \left(\min _{i=1}^{p}\left|s(\mathbf{x})-s_{i}\right|\right)
$$

Once computed the codified patterns, the de-codifying patterns and $s_{k}$ we can build the associative memory.

Let $\left\{\left(\overline{\mathbf{x}}^{k}, \overline{\mathbf{y}}^{k}\right) \mid k=1, \ldots, p\right\}, \overline{\mathbf{x}}^{k} \in \mathbf{R}^{n}, \overline{\mathbf{y}}^{k} \in \mathbf{R}^{m}$ a fundamental set of associations (codified patterns). Synapses of associative memory $\mathbf{W}$ are defined as:

$$
w_{i j}=\bar{y}_{i}-\bar{x}_{j}
$$

After computed the codified patterns, the de-codifying patterns, the reader can easily corroborate that any association can be used to compute the synapses of $\mathbf{W}$ without modifying the results. In short, building of the associative memory can be performed in three stages as:

1. Transform the fundamental set of association into codified and decodifying patterns by means of previously described Procedure 1.

2. Compute simplified versions of input patterns by using equation 1.

3. Build $\mathbf{W}$ in terms of codified patterns by using equation 3 .

\subsection{Modifying Synapses of the Associative Model}

As we had already mentioned, synapses could change in response to an input stimulus; but which synapses should be modified? For example, a head injury might cause a brain lesion killing hundred of neurons; this entails some synapses to reconnect with others neurons. This reconnection or modification of the synapses might cause that information allocated on brain will be preserved or will be lost, the reader could find more details concerning to this topic in [5] and [12]. 
This fact suggests there are synapses that can be drastically modified and they do not alter the behavior of the associative memory. In the contrary, there are synapses that only can be slightly modified to do not alter the behavior of the associative memory; we call this set of synapses the kernel of the associative memory and it is denoted by $\mathbf{K}_{\mathbf{W}}$. In the model we find two types of synapses: synapses that can be modified and do not alter the behavior of the associative memory; and synapses belonging to the kernel of the associative memory. These last synapses play an important role in recalling patterns altered by some kind of noise.

Let $\mathbf{K}_{\mathbf{W}} \in \mathbf{R}^{n}$ the kernel of an associative memory $\mathbf{W}$. A component of vector $\mathbf{K}_{\mathbf{W}}$ is defined as:

$$
k w_{i}=\operatorname{mid}\left(w_{i j}\right), j=1, \ldots, m
$$

According to the original idea of our proposal, synapses that belong to $\mathbf{K}_{\mathbf{W}}$ are modified as a response to an input stimulus. Input patterns stimulate some ARs, interact with these regions and then, according to those interactions, the corresponding synapses are modified. Synapses belonging to $\mathbf{K}_{\mathbf{W}}$ are modified according to the stimulus generated by the input pattern. This adjusting factor is denoted by $\Delta w$ and could be estimated as:

$$
\Delta w=\Delta(\mathbf{x})=s\left(\overline{\mathbf{x}}^{a r}\right)-s(\mathbf{x})
$$

where $a r$ is the index of the AR.

Finally, synapses belonging to $\mathbf{K}_{\mathbf{W}}$ are modified as:

$$
\mathbf{K}_{\mathbf{W}}=\mathbf{K}_{\mathbf{W}} \oplus\left(\Delta w-\Delta w_{\text {old }}\right)
$$

where operator $\oplus$ is defined as $\mathbf{x} \oplus e=x_{i}+e \forall i=1, \ldots, m$. As you can appreciate, modification of $\mathbf{K}_{\mathbf{W}}$ in equation 6 depends of the previous value of $\Delta w$ denoted by $\Delta w_{\text {old }}$ obtained with the previous input pattern. Once trained the DAM, when it is used by first time, the value of $\Delta w_{\text {old }}$ is set to zero.

\subsection{Recalling a Pattern Using the Proposed Model}

Once synapses of the associative memory have been modified in response to an input pattern, every component of vector $\overline{\mathbf{y}}$ can be recalled by using its corresponding input vector $\overline{\mathbf{x}}$ as:

$$
\bar{y}_{i}=\operatorname{mid}\left(w_{i j}+\bar{x}_{j}\right), j=1, \ldots, n
$$

In short, pattern $\overline{\mathbf{y}}$ can be recalled by using its corresponding key vector $\overline{\mathbf{x}}$ or $\tilde{\mathbf{x}}$ in six stages as follows:

1. Obtain index of the active region $a r$ by using equation 2 .

2. Transform $\mathbf{x}^{k}$ using de-codifying pattern $\hat{\mathbf{x}}^{a r}$ by applying the following transformation: $\widehat{\mathbf{x}}^{k}=\mathbf{x}^{k}+\hat{\mathbf{x}}^{a r}$.

3. Estimate adjusting factor $\Delta w=\Delta(\hat{\mathbf{x}})$ by using equation 5 . 
4. Modify synapses of associative memory $\mathbf{W}$ that belong to $\mathbf{K}_{\mathbf{W}}$ by using equation 6 .

5. Recall pattern $\hat{\mathbf{y}}^{k}$ by using equation 7 .

6. Obtain $\mathbf{y}^{k}$ by transforming $\widehat{\mathbf{y}}^{k}$ using de-codifying pattern $\hat{\mathbf{y}}^{a r}$ by applying transformation: $\mathbf{y}^{k}=\widehat{\mathbf{y}}^{k}-\hat{\mathbf{y}}^{a r}$.

The formal set of prepositions that support the correct functioning of this dynamic model and the main advantages against other classical models can be found in [19], interesting applications of this model are described in [14] and [15].

In general, we distinguish two main parts for the model: the active region part (PAR) which determines the active region; and the pattern recall part (PPR). PAR (first step during recall procedure) sends a signal to PPR (remaining steps for recall procedure). This indicates the region activated by the input pattern. A schematic figure of this model is shown in Fig. 1(a).

\section{Proposal}

As we have previously said, baby's brain can differentiate faces from other faces within a few months of birth. However, they are not capable of detecting subtle features in a face. This fact could suggest the baby uses only few information of the face in order to recognize it.

When babies perceive a scene, we conjecture that babies at random select some stimulating points SPs, and then these information passes through the brain to the visual cortex, where this information is analyzed. Finally the information passes to other regions of the brain involved in learning and recognition processes.

In order to recognize different images of faces we propose to use a network of associative memories nDAMs. Then, we divide the image in different parts (subpatterns) and over each sub-pattern, we detect subtle features by means of a random selection of SPs. At last, each DAM of the $\mathrm{nDAM}$ is fed with these sub-patterns for training and recognition.

\subsection{Random Selection Using a Modified DAM}

In the DAM model, the simplified version of an input pattern is the middle value of input pattern. This value is computed by using mid operator. In order to simulate the random selection of the infant vision system we substitute mid operator with rand operator defined as follows:

$$
\text { rand } \mathbf{x}=x_{s p}
$$

where $s p=\operatorname{random}(n)$ is a random number between zero and the length of input pattern. This represents a stimulation point. $s p$ is a constant value computed at the beginning of the building phase. During recalling phase $s p$ takes the same value. rand operator uses a uniform random generator to select a component over each part of the pattern. We adopt this operator based on the hypothetical idea about infants are interested into sets of features where each set is different with some intersection 
among them. By selecting features at random, we conjecture that at least we select a feature belonging to these sets.

\subsection{Implementation of the Proposal}

During recalling, each DAM recovers a part of the image based on the AR of each DAM. However, a part of the image could be wrongly recalled because its corresponding AR could be wrongly determined due to some patterns do not satisfy the prepositions that guarantee perfect recall. To avoid this, we use an integrator. Each DAM determines an AR, the index of the AR is sent to the integrator, the integrator determines which was the most voted region and sends to the DAMs the index of the most voted region (the new AR). In Fig. 1(b) a general architecture of the network of DAMs is shown.

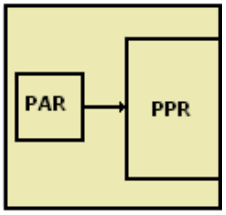

(a)

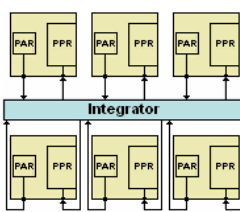

(b)

Fig. 1. (a) Main parts of the DAM. (b) Architecture of a network of DAMs.

In order to interconnect several DAMs we disconnect the PAR of its corresponding PPR. The output of the PAR is sent to an external integrator. This integrator receives several signals from different DAMs indicating the AR. At last, based on a voting technique, the integrator "tells" to each DAM which is the most voted AR.

Schematic representation of building and recalling phase are show in Fig. 2. Building of the nDAMs is done as follows:

Let $\left[\mathbf{I}_{x}^{k}\right]_{a \times b}$ and $\left[\mathbf{I}_{y}^{k}\right]_{c \times d}$ an association of images and $r$ be the number of DAMs.

1. Transform the images into a vector $\left(\mathbf{x}^{k}, \mathbf{y}^{k}\right)$ by means of the standard image scan method where vectors are of size $a \times b$ and $c \times d$ respectively.

2. Decompose $\mathbf{x}^{k}$ and $\mathbf{y}^{k}$ in $r$ sub-patterns of the same size.

3. Take each sub-pattern (from the first one to the last one $(r)$ ), then take at random a stimulating point $s p_{i}, i=1, \ldots, r$ and extract the value at that position.

4. Train $r$ DAMS as in building procedure taking each sub-pattern (from the first one to the last one $(r)$ ) using rand operator.

Pattern $\mathbf{I}_{y}^{k}$ can be recalled by using its corresponding key image $\mathbf{I}_{x}^{k}$ or distorted version $\tilde{\mathbf{I}}_{x}^{k}$ as follows:

1. Transform the images into a vector by means of the standard image scan method. 
2. Decompose $\mathbf{x}^{k}$ in $r$ sub-patterns of the same size.

3. Use the stimulating point, $s p_{i}, i=1, \ldots, r$ computed during the building phase and extract the value of each sub-pattern.

4. Determine the most voted active region using the integrator.

5. Substitute mid operator with rand operator in recalling procedure and apply steps from two to six as described in recalling procedure on each memory.

6. Finally, put together recalled sub-patterns to form the output pattern.

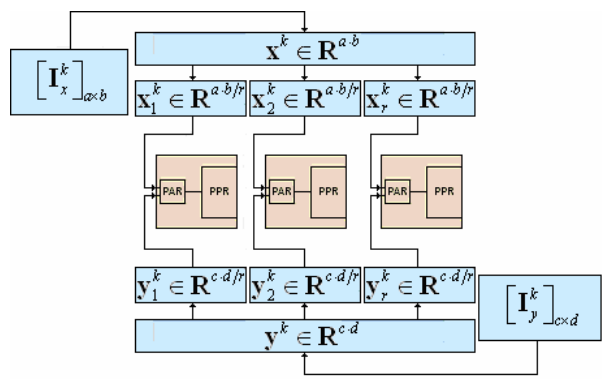

(a)

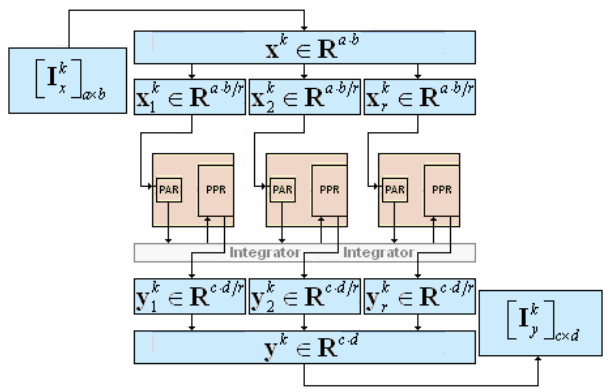

(b)

Fig. 2. (a) Schematic representation of building phase. (b) Schematic representation of the recalling phase.

While PCA dimension reduction techniques require the covariance matrix to build an Eigenspace, and then project patterns using this space to eliminate redundant information, our proposal only requires a random selection of stimulating points. This approach contributes to eliminating redundant information, it is less computationally expensive than PCA, and helps the nDAMs or other classification tools to learn efficiently the faces.

The main reason to use a DAM (in our proposal) is to demonstrate that, as in the case of a neural network and other classifiers, DAMs can be used to solve complex problems. To our acknowledgment, nobody had used associative memories this way for face recognition. 


\section{Experimental Results}

We tested the efficiency of the proposal with the benchmark of faces given in [4]. This database contains twenty photos of fifteen different people. Each photo is in colour and of $180 \times 200$ pixels. Refer to Fig. 3. Furthermore, people in images appear with different gesticulations which nowadays is still a challenge in face recognition. Due to the level of complexity of this benchmark, we decided to use it to test the accuracy of the proposal. The database of images was divided into two sets of images. First photo of each person (15 in total) was used to train the network of DAMs. The remaining 285 photos (19 for each person) were used to test the efficiency of the proposal.

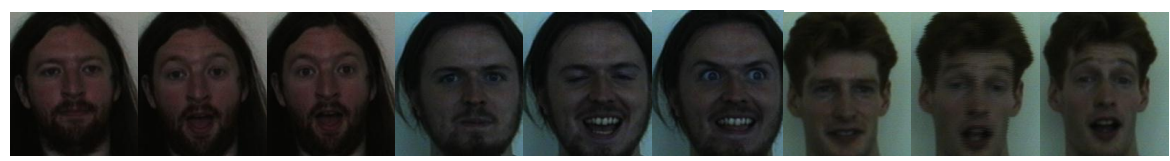

(a)

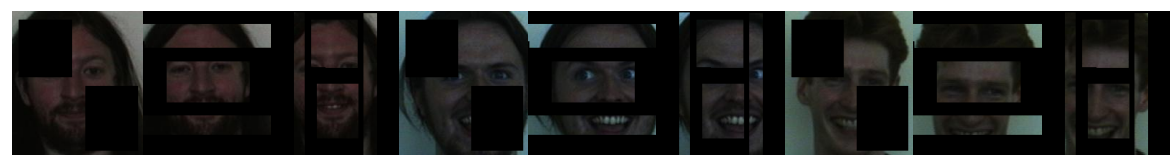

(b)

Fig. 3. (a) First 3 images of the photos of 3 people of the 15 used to test the efficiency of the proposal. (b) First 3 images of the photos the 3 people of the 15 used to test the efficiency of the proposal in the presence of occlusions.

To train the nDAM, only one image of the fifteen face classes were used along with the building procedure described in section 4.2. Something important to remark is that each DAM belonging to the $\mathrm{nDAM}$ was trained into its auto-associative version, i.e. $\mathbf{I}_{x}^{k}=\mathbf{I}_{y}^{k}$. During recalling phase the second set of images and the recalling procedure described in section 4.2 were used.

The accuracy of the proposal was tested using several configurations, starting with networks composed by one DAM until 1100 DAMs. Because of stimulating points (pixels) were randomly selected, we decided to test the stability of proposal with the same configuration 20 times.

As you can appreciate from Fig. 4(a), the accuracy of the proposal increases when the number of stimulation points (or the number of DAM) also increases. In Fig. 4(a), we can appreciate that after a certain number of stimulating points the accuracy of the proposal tends to be stable. In general, the results obtained in these experiments were around $99 \%$ of recognition.

In addition, the accuracy of the proposal was tested when the testing images were partially occluded. For this, manually one or more parts of each image (of the second set of images) were occluded with regions of different forms and sizes. Figure 3(b) shows the first three images of three people used for testing.

In the previous experiments, the stimulating points were also randomly selected. As you can appreciate from Fig. 4(b), the accuracy of the proposal increases as the 
number of stimulating points (or the number of DAM) is increased. In Fig. 4(b), we can appreciate that after a certain number of stimulating points the accuracy of the proposal tends to stabilize. In general, the results obtained in these experiments were around $80 \%$ of recognition.

We have tested the efficiency of the proposal. We have verified that the worst performance was obtained with the images in presence of occlusion. The results obtained with the proposal in the first set of experiments were comparable with those obtained by means of a PCA-based method (99\% of accuracy). Although PCA is a powerful technique it consumes a lot of time to reduce the dimensionality of the data. Our proposal, because of its simplicity in operations, is not a computationally expensive technique and the results obtained are comparable to those provided by PCA.

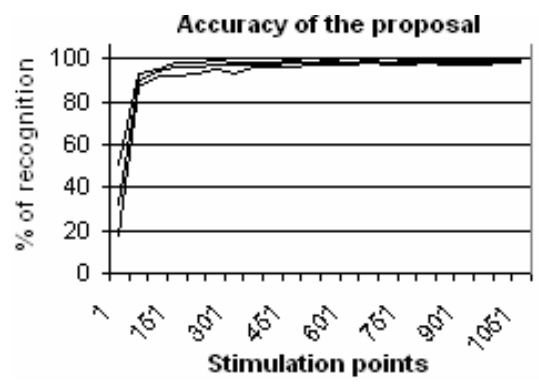

(a)

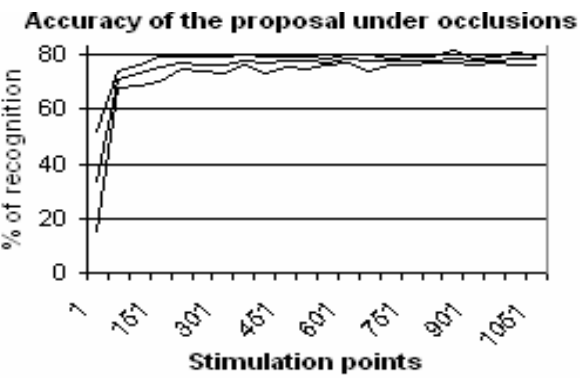

(b)

Fig. 4. (a) Behavior of the proposal. (b) Behavior of the proposal in the presence of occlusions. Maximum, average and minimum accuracy are sketched.

\section{Conclusions}

In this work we have shown that by applying some aspects of the infant vision system it is possible to enhance some associative memories and also make possible its application to complex problems such as face recognition.

Based on some biological aspects of the infant vision system, we have proposed some modifications to the DAM. In addition, we have described the algorithms for training and operation of a network of DAMs.

Through several experiments we have shown the accuracy and the stability of the proposal even in the presence of occlusions. In average the accuracy of the proposal oscillates between $96 \%$ and $99 \%$. Important to mention is that, to our knowledge, nobody has reported results of this type using an associative memory for face recognition.

The results obtained with the proposal were comparable with those obtained by means of a PCA-based method. Although PCA is a powerful technique it consumes a lot of time to reduce the dimensionality of the data. Our proposal, because of its simplicity in operations, is not a computationally expensive technique and the results obtained are comparable to those provided by PCA.

Nowadays, we are applying these ideas in $3 \mathrm{D}$ object recognition and image retrieval taking into account some other aspects of the vision system. 
Acknowledgments. This work was economically supported by SIP-IPN under grant 20071438 and CONACYT under grant 46805.

\section{References}

[1] Hopfield, J.J.: Neural networks and physical systems with emergent collective computational abilities. Proc. of the Nat. Academy of Sciences 79, 2554-2558 (1982)

[2] Jolliffe, I.: Principal Component Analysis. Springer, Heidelberg (1986)

[3] Kutas, M., Hillyard, S.A.: Brain potentials during reading reflect word expectancy and semantic association. Nature 307, 161-163 (1984)

[4] Spacek, L.: Collection of facial images: Grimace (1996), Available from http://cswww.essex.ac.uk/ mv/ allfaces/grimace.html

[5] Reinvan, I.: Amnestic disorders and their role in cognitive theory. Scandinavian Journal of Psychology 39(3), 141-143 (1998)

[6] Price, C.J.: The anatomy of language: contributions from functional neuroimaging. Journal of Anatomy 197(3), 335-359 (2000)

[7] Sussner, P.: Generalizing operations of binary auto-associative morphological memories using fuzzy set theory. Journal of math. Imaging and Vision 19(2), 81-93 (2003)

[8] Laughlin, S.B., Sejnowski, T.J.: Communication in neuronal networks. Science 301, 1870-1874 (2003)

[9] Sossa, H., Barron, R.: New associative model for pattern recall in the presence of mixed noise. In: Proc. of the fifth IASTED-SIP2003, vol. 399, pp. 485-490. Acta Press (2003)

[10] Sossa, H., Barrón, R., Vázquez, R.A.: Transforming Fundamental set of Patterns to a Canonical Form to Improve Pattern Recall. In: Lemaître, C., Reyes, C.A., González, J.A. (eds.) IBERAMIA 2004. LNCS (LNAI), vol. 3315, pp. 687-696. Springer, Heidelberg (2004)

[11] Slaughter, V., Stone, V.E., Reed, C.: Perception of Faces and Bodies Similar or Different? Current Directions in Psychological Science 13(9), 219-223 (2004)

[12] Jovanova-Nesic, K., Jankovic, B.D.: The Neuronal and Immune Memory Systems as Supervisors of Neural Plasticity and Aging of the Brainn: From Phenomenology to Coding of Information. Annals of the New York Acad.of Sci. 1057, 279-295 (2005)

[13] Cuevas, K., et al.: Infants Form Associations Between Memory Representations of Stimuli That Are Absent. Psychological Science 17(6), 543-549 (2006)

[14] Vazquez, R.A., Sossa, H.: Associative Memories Applied to Image Categorization. In: Martínez-Trinidad, J.F., Carrasco Ochoa, J.A., Kittler, J. (eds.) CIARP 2006. LNCS, vol. 4225, pp. 549-558. Springer, Heidelberg (2006)

[15] Vazquez, R.A., Sossa, H., Garro, B.A.: A New Bi-directional Associative Memory. In: Gelbukh, A., Reyes-Garcia, C.A. (eds.) MICAI 2006. LNCS (LNAI), vol. 4293, pp. 367380. Springer, Heidelberg (2006)

[16] Sussner, P., Valle, M.: Implicative Fuzzy Associative Memories. IEEE Trans. on Fuzzy Systems 14(6), 793-807 (2006)

[17] Sussner, P., Valle, M.: Gray-Scale Morphological Associative Memories. IEEE Trans. on Neural Networks 17(3), 559-570 (2006)

[18] Sossa, H., Barron, R., Vazquez, R.A.: Study of the Influence of Noise in the Values of a Median Associative Memory. In: Beliczynski, B., et al. (eds.) ICANNGA 2007, Part II, LNCS, vol. 4432, pp. 55-62. Springer, Heidelberg (2007)

[19] Vazquez, R.A., Sossa, H.: A new associative memory with dynamical synapses (to be submitted, 2007) 